\title{
Ensino médico e as controvérsias da realidade no rastreamento inicial do câncer de mama na mulher
}

\section{Medical Education and Reality Controversies in Initial Breast Cancer Screening in woman}

\author{
Guilherme de Almeida Bastos ${ }^{1}$ \\ Alex Monteiro Leal da Paixão ${ }^{2}$ \\ Evandro Andrade Silva ${ }^{3}$ \\ Tatiana Vieira Tolentino ${ }^{4}$ \\ Márcia Ribeiro Braz ${ }^{5}$ \\ Adilson da Costa Filho ${ }^{6}$
}

\section{Resumo}

O ensino médico de ginecologia deve orientar os seus discentes para a importância da mamografia no rastreamento do câncer de mama da mulher, porque este método diagnóstico tem sido considerado o exame mais sensível para a detecção precoce deste tipo de câncer. O programa Viva Mulher do Ministério da Saúde, é um programa multidisciplinar de aconselhamento genético, estabelecendo um cenário ideal para orientação das famílias de alto risco e com protocolo de pesquisa coordenados pelo corpo docente, e poderá ser dimensionado para o ensino médico. Uma vez que a lei estabelece a realização da mamografia a partir dos 40 anos nas mulheres, como método de rasteamento inicial do câncer de mama, sem, no entanto, especificar o tempo em anos da repetição do exame, o profissional fica exposto a questóes éticas e aos processos judiciais inerentes aos atendimentos desses pacientes do Sistema Único de Saúde (SUS), realizados nos ambulatórios. É necessário dentro da academia, o levantamento e discussão desta questão, para que o futuro médico tenha sua base de atendimento e experiência consolidados ainda no curso de Graduação em Medicina e apto para o mercado de trabalho neste setor.

Palavras-chave: ensino médico, mamografia, câncer de mama, legislação.

\begin{abstract}
Medical teaching of gynecology should guide their students to the importance of mammography in breast cancer screening of women, this diagnostic method has been considered the most sensitive exam for early detection of cancer. The program Viva Mulher of the Ministry of Health is a multidisciplinary program of genetic counseling, establishing the guidance of high-risk families and a research protocol coordinated the researchers, may be sized for medical education. Since the law provides mammography for 40 year old women without, however, specifying the time in years re-examination, the practitioner is exposed to ethical and legal proceedings relating to the care of these patients Health System (SUS), conducted in outpatient clinics. It is necessary to an extensive discussion on this matter within the Board of Medicine as well as within the academy so that the physician be aware that the option of using this diagnostic method after fifty years of age will be based on the rigors of law.
\end{abstract}

Keywords: Medical Education, Mammography, Breast Cancer, Legislation.

\footnotetext{
1,2,3 e 4 Mestrandos do Programa de Mestrado em Ensino em Ciências da Saúde e do Meio Ambiente /UniFOA.
}

${ }^{5}$ Doutora em Enfermagem/UFRJ. Orientadora do Programa de Mestrado em Ensino em Ciências da Saúde e do Meio Ambiente/UniFOA.

${ }^{6}$ Doutor em Química Biológica (Bioquímica Médica)/UFRJ. Orientador do Prog. Mestrado em Ensino de Ciências da Saúde e do Meio Ambiente/UniFOA. 


\section{INTRODUÇÃO}

Atualmente há um consenso entre especialistas em educação médica que uma das competências mais importantes a serem adquiridas durante o curso de graduação é a capacidade de se atualizar continuamente, de saber como buscar a informação que é fundamental para resolver algum problema clínico, e saber selecionar a informação relevante e utilizar os dados obtidos de forma crítica. Hoje é impossível que todos os conteúdos de cada área da Medicina sejam transmitidos ao estudante durante seu curso. Os docentes de Medicina têm a tarefa de selecionar o que é realmente importante de sua área para a formação geral de todos os médicos.

Nesse sentido, o ensino médico de ginecologia deve orientar os seus discentes para a importância da mamografia no rastreamento do câncer de mama da mulher, porque este método diagnóstico tem sido considerado o exame mais sensível para a detecção precoce deste tipo de câncer. O programa Viva Mulher do Ministério da Saúde, é um programa multidisciplinar de aconselhamento genético, estabelecendo um cenário ideal para orientação das famílias de alto risco e com protocolo de pesquisa coordenados pelo corpo docente, poderá ser dimensionado para o ensino médico. Uma vez que a lei estabelece a realização da mamografia a partir dos 40 anos nas mulheres, como método de rasteamento inicial do câncer de mama, sem, no entanto, especificar o tempo em anos da repetição do exame, o profissional fica exposto a questóes éticas e aos processos judiciais inerentes aos atendimentos desses paciente do Sistema Único de Saúde (SUS), realizados nos ambulatórios.

É necessário dentro da academia, o levantamento e discussão desta questão, para que o futuro médico tenha sua base de atendimento e experiência consolidados ainda no curso de Graduação em Medicina e apto para o mercado de trabalho neste setor.

O câncer de mama é provavelmente, o mais temido pelas mulheres, devido à sua alta incidência e aos efeitos psicológicos sobre a percepção da sexualidade e a sua imagem pessoal. A cada ano, no mundo são registrados mais de 180 mil casos novos e 40 mil óbitos por câncer de mama (REZENDE, et al., 2008).

Dados da Câncer Statistics aponta em 2008, 182.460 novos casos de câncer de mama em mulheres nos Estados Unidos, e 40.480 mortes em decorrência dele (JEMAL et al., 2008 apud HUGUET et al., 2009). De acordo com dados do Instituto Nacional do Câncer (INCA), em estimativa feita para 2008, serão diagnosticados no Brasil 49.470 casos novos, sendo a segunda neoplasia mais incidente em mulheres (BRASIL, 2008). Com o aumento das taxas de detecção precoce e com a melhoria nos tratamentos oferecidos, sabese que aproximadamente $50 \%$ das mulheres sobreviverão por, pelo menos, quinze anos após o diagnóstico e deverão ajustar-se às seqüelas cirúrgicas. Estatísticas mundiais indicam que uma em nove mulheres irá desenvolver câncer mamário durante as suas vidas (HUGUET et al., 2009).

De acordo com Brito et al. (2010), o câncer de mama se constitui na primeira causa de morte por câncer entre as mulheres. $\mathrm{O}$ autor prossegue dizendo que no Brasil, o câncer de mama é o mais incidente em todas as regióes, exceto na regiáo Norte, na qual perde para o câncer de colo uterino, segundo dados do INCA, que estimou, para todo o Brasil, aproximadamente 49.240 casos novos em 2010.

Não existe nenhum método isento de falhas como prevenção primária para o câncer de mama; porém, existem três estratégias de prevenção secundária para a detecção precoce: o autoexame das mamas (AEM), o exame clínico das mamas (ECM) e a mamografia bilateral (MMG), sendo esta última considerada o método de eleição de rastreio em programas populacionais pelo seu impacto sobre a mortalidade (BRITO et al., 2010).

Em todo mundo, os resultados clínicos do câncer de mama têm melhorado, graças ao aumento do número de casos detectados em estágio mais precoce, com o uso da mamografia como exame de rastreamento, otimizando-se o tratamento cirúrgico, a radioterapia e o desenvolvimento de tratamentos mais ordenados (FÉLIX et al., 2010).

\section{DISCUSSÃO}

O Ministério da Saúde (MS) considerando a situação do câncer de mama no Brasil realizou um trabalho conjunto entre o INCA e a Área Técnica da Saúde da Mulher, com o apoio da Sociedade Brasileira de Mastologia, nos dias 13 e 14 de novembro de 2003. Uma oficina de trabalhos para discussão e aprovação de recomendaçóes referentes ao controle do câncer de mama. Este documento de consenso apresentava as recomendações para a prevenção, detecção precoce, diagnóstico, tratamento e cuidados paliativos no câncer de mama e estratégias que deveriam ser utilizada para sua implementação no Sistema Único de Saúde (BRASIL, 2004)

Para Marchi et al. (2010) as diretrizes no Brasil são conflitantes, pois recomenda-se o rastreamento mamográfico bienal para mulheres não pertencentes a grupos populacionais de risco, somente a partir de 50 anos de idade. No setor suplementar de assistência à saúde, defende-se a triagem mamográfica anual a partir de 40 anos, bienalmente entre 50 e 69 anos e, de acordo com a expectativa de vida da mulher, após os 70 de idade.

No Brasil, o aumento da mortalidade por câncer de mama tem sido atribuído, sobretudo, ao retardamento no diagnóstico e na implementação da terapêutica adequada. $\mathrm{O}$ 
MS vem recomendando desde 2004, a realizaçáo de mamografia de rastreamento para mulheres assintomáticas, na faixa etária entre 50 e 69 anos, com intervalos nunca superiores há dois anos. Já para mulheres entre 40 e 49 anos de idade, a recomendaçáo sendo de rastreamento anual por meio do exame clínico da mama (BRASIL, 2004). Contudo, a partir de 29 de abril de 2009, o Sistema Único de Saúde (SUS) deverá assegurar realização de exame mamográfico a todas as mulheres a partir dos 40 anos de idade, conforme estabelece a lei número 11.664 , de 29 de abril de 2008. O SUS, responsável pela assistência médica de cerca de $70 \%$ dos brasileiros, realizou, entre 2005 e 2007, em média 2,6 milhóes de mamografias a cada ano, o que corresponde a $11 \%$ do número de mulheres entre 40 e 69 anos de idade. (BRASIL, 2009).

$\mathrm{O}$ auto-exame das mamas tem sido objeto de várias campanhas preventivas e publicitárias em todo mundo para prevenção do câncer de mama e ainda há pouca evidência de sua eficácia. Os dois maiores estudos controlados existentes não mostraram reduçáo do tamanho do tumor nem diminuição das metástases axilares e houve número maior de biópsias desnecessárias no grupo de mulheres que faziam auto-exame. $\mathrm{O}$ auto-exame também serve para a difusão de informaçōes sobre o câncer de mama e para que a mulher possa conhecer melhor o seu corpo (PAULINELLI et al., 2004).

O exame clínico é outra alternativa para o diagnóstico de câncer de mama. Um profissional treinado pode encontrar nódulos menores que $1 \mathrm{~cm}$. $\mathrm{O}$ valor do exame clínico para a reduçáo da mortalidade para o câncer de mama também é discutível (JATOI et al., 1999 apud PAULINELLI et al., 2004).

De acordo com as New Recommendations From The United States Govermente On Breast Câncer Screening, a mamografia é método reconhecidamente eficaz na redução da mortalidade por câncer de mama, em especial na pósmenopausa. Estima-se que a sensibilidade da mamografia em programas de rastreamento esteja entre 77 e $95 \%$, diminuindo para 51 a $83 \%$ em mulheres com menos de 50 anos, com mamas densas ou em uso de terapia hormonal (PAULINELLI et al., 2004).

A sensibilidade da mamografia está ainda muito aquém do ideal, especialmente em mulheres jovens e com mamas densas. A mamografia apresenta índice de falso-positivos e falsonegativos de aproximadamente $10 \%$ (JUNIOR et al., 2006).

De acordo com a literatura a mamografia tem sensibilidade entre $88 \%$ e $93,1 \%$ e especificidade entre $85 \%$ e $94,2 \%$, e a utilização desse exame como método de rastreamento reduz a mortalidade em 25\% (INCA 2010).

Outro método de grande importância no diagnóstico do câncer de mama, é a ultrassonografia, embora ela não seja considerada como método de rastreamento. A ultrassonografia tem pouco a oferecer em mulheres com mamas lipossubstituidas e oferece maior risco de falso-negativo; mas é de grande utilidade em mamas densas, em que a sensibilidade da mamografia fica muito prejudicada. A adição da ultrassonografia à mamografia diminui também substancialmente o número de biópsias por alteraçōes benigna. A ultrassonografia é uma modalidade de imagem utilizada na avaliação de lesôes mamárias para distingui-las entre sólidas e líquidas. Não sendo recomendado como método primário na detecção do câncer de mama por conta da alta variabilidade da taxa de falso-negativos, que vai de 0,3 a $47 \%$ (FIALHO et al., 2008).

As imagens adquiridas por ressonância magnética (RM), que foram iniciadas na década de 80 , para detecção do câncer de mama precoce, com uma sensibilidade entre 95 e 100\%, falso negativos, e especificidade entre 37 e $97 \%$, falso positivos. A RM é um método de alto custo, limitado em demonstrar calcificaçóes e muito sensível em mamas densas.Este método tem a capacidade de detectar cânceres adicionais, doença multifocal, náo percebidos à mamografia e a ultrassonografia mamária e em pacientes já submetidas à radioterapia, na qual incluem espessamento da pele, edema, cicatrizes e distorçóes arquiteturais da mama e mudar consequentemente o estadiamento e o plano cirúrgico (FIALHO et al., 2008).

A cintilografia é um método de diagnóstico por imagem utilizada principalmente para detecçáo do linfonodo sentinela e seu uso como rastreamento primário também não é justificado, pelo alto custo, visto que sua sensibilidade tem taxas discretamente superiores às da mamografia (FIALHO et al., 2008).

Com a maior aceitação do rastreio mamográfico, estudos têm demonstrado redução na mortalidade de aproximadamente $24 \%$ nas mulheres entre 40 e 49 anos e de $30 \%$ nas pacientes com mais de 50 anos, submetidas a esse programa. (RODRIGUES et al., 2008).

A idade, na ocasião do diagnóstico, também é descrita como fator preditivo de maior risco. De acordo com uma revisão européia com mais de 1.700 casos, o risco relativo para desenvolvimento de metástases durante o seguimento foi mais de duas vezes maior em pacientes com menos de 40 anos, em comparação com pacientes mais velhas (CLAGNAN et al., 2008).

A mamografia é ainda hoje, o melhor método de detecção precoce do câncer da mama, sendo a interpretração de uma mamografia um desafio para o especialista. A mamografia digital foi desenvolvida, em parte, com o objetivo de compensar algumas das limitaçôes da mamografia analógica. Na mamografia digital, os processos de aquisição, exposiçáo e armazenamento das imagens são separados, permitindose a otimização de cada um. O processamento das imagens digitais permite que a intensidade do contraste de cada imagem seja manipulada, possibilitando que áreas mais densas 
da mama tenham contraste mais intenso. Sendo importante ressaltar que nenhum dos dois métodos foi infalível na detecção das lesôes malignas (RODRIGUES et al., 2008).

De acordo com Amaral (2010), o exemplo típico é que a quantidade de mamografias não aumentou mesmo com maior número de mamógrafos em funcionamento no país. Este autor comenta que foi realizada uma auditoria realizada pelo Tribunal de Contas da União que mostrou a subutilizaçáo da maioria dos mamógrafos operantes em serviços públicos no Brasil entre maio de 2008 e abril de 2009. Em apenas 23 locais (de 435), foram feitas 25 mamografias ou mais por dia, parâmetro considerado ideal pelos técnicos do Tribunal. A média nacional de produção das máquinas foi 9,8 exames/dia.

O Viva Mulher - Programa Nacional de Controle do Câncer de Colo do Útero e de Mama (BRASIL, 2001) tem como diretrizes: articular e integrar uma rede nacional, motivar a mulher a cuidar de sua saúde, reduzir a desigualdade de acesso da mulher a rede de saúde, melhorar a qualidade do atendimento a mulher e aumentar a eficiência da rede de controle do câncer. Neste Programa, o rastreamento de mulheres assintomáticas por mamografia deve ser realizado entre 50 a 69 anos de idade, com intervalo máximo de dois anos entre os exames.

A mamografia de rastreamento tem sido considerada o teste mais sensível para a detecção precoce do câncer de mama não palpável. $\mathrm{O}$ estudo histopatológico de biópsias mamárias de pacientes portadoras de mamografias contendo microcalcificaçôes suspeitas revelou câncer de mama em ate $30 \%$ dos casos, sendo que o agrupamento de microcalcificaçôes heterogêneas e pleomórficas, encontradas no estudo mamográfico, foi representativo de câncer in situ em até 90\% dos casos (RESENDE et al.,2008).

As calcificações vasculares mamárias é um achado freqüente nas mamografias e não devem ser ignoradas, sendo vistas apenas como uma alteração benigna sem qualquer outro significado. Devendo-se orientar estas pacientes quanto à prevenção primária das doenças cardiovasculares e diabetes (OLIVEIRA et al., 2009).

Para o Programa "Viva Mulher", mulheres que apresentam fatores de risco para o desenvolvimento do câncer de mama, como: menarca precoce, primípara idosa, câncer de ovário ou de mama anterior, doença mamária benigna, alta densidade mamária, mutaçôes genéticas, histórico familiar de câncer de mama, têm que realizar o rastreamento do câncer mamário anualmente a partir dos 35 anos de idade. O governo garante o acesso ao diagnóstico do câncer de mama, tratamento e seguimento para todas as mulheres com alteraçôes nos exames realizados (BRASIL, 2001).

A grande controvérsia médica e legal, surgiu a partir do momento em que a lei $n^{\circ} 11,664 / 08 /$ lei no 11,664 de 29 de abril de 2008, no seu artigo $2^{\circ}$ inciso III estabelece: "a realização de exame mamográfico a todas as mulheres a partir dos 40 quarenta anos de idade”. Percebe-se que não há restriçóes a realização da mamografia por mulheres que não pertençam ao grupo de risco e, esta Lei, também não especifica a periodicidade em que o médico deve solicitar este exame.

A Constituição Federal do Brasil (BRASIL, 1988), em seu artigo 5० inciso II diz: "Ninguém será obrigado a fazer ou deixar de fazer alguma coisa senão em virtude da lei”. Assim, como o novo Código de Ética Médica, em vigor desde 13 abril de 2010 em seu capítulo III, artigo $1^{\circ}$ - parágrafo único afirma que: "A responsabilidade médica é sempre pessoal e não pode ser presumida" (NEVES e SIQUEIRA, 2010). No entanto, a justiça entende que é uma relação de consumo e que os pacientes podem acionar os profissionais da saúde com gratuidade de justiça, sem direito de reversão pelos mesmos. Esses pacientes ainda encontram alguns advogados inescrupulosos que podem tirar proveito dos médicos, sujeitos a adversidades inerentes de sua profissão, muitas vezes, por não ter solicitado a mamografia a partir dos 40 anos de idade e que por um indiciamento judicial, torna-se um pesadelo, uma punição não merecida caso haja uma conscientização imparcial pelo juiz.

O Programa de Viva Mulher que já vinha sendo implementado pelo Governo Federal, no qual se aplica a mamografia para as mulheres a partir dos 50 anos entra em confronto com a Lei 11.664/08, a qual ordena o exame de mamografia para as mulheres a partir dos 40 anos. Há de convir que um Programa de Saúde tem força administrativa e a lei é imperativa e se impóe a todos. Ninguém a pode ignorar conforme os termos do Art. $3^{\circ}$ da Lei de Introdução ao Código Civil, 2002 (MECUM, 2009). Assim, depreende-se do entendimento de que o melhor será cumprir a lei ordinária que é o Código Civil, sob pena de arcar com a Responsabilidade Civil, conforme abordado anteriormente além de poder ser responsabilizado criminalmente também na forma da Lei Penal. A melhor alternativa para o profissional da medicina é cumprir a lei para as mulheres dos 40 aos 50 anos e a partir dos 50 anos de idade, esse profissional estará cumprindo cumulativamente a lei e o Programa de Saúde do SUS.

\section{FERRAMENTA DE ENSINO NO INTERNATO NA DISCIPLINA DE GINECOLOGIA}

Galileu Galilei (1564-1642) afirmou que: "não se pode ensinar coisa alguma a alguém, pode-se apenas auxiliá-la a descobrir por si só" (CARVALHO 2009).

$\mathrm{O}$ ensino médico de ginecologia na graduação deverá orientar os seus discentes para a importância da mamografia 
no rastreamento do câncer inicial da mama na mulher, porque este método diagnóstico tem sido considerado o exame mais sensível para a detecção precoce deste tipo de patologia. O Viva Mulher do Ministério da Saúde, é um programa multidisciplinar de aconselhamento genético, estabelecendo um cenário ideal para orientação das famílias de alto risco e com protocolo de pesquisa coordenados pelo corpo docente, poderá ser dimensionado. Uma vez que a lei no 11,664/08/ lei no 11,664 de 29 de abril de 2008 estabelece a realização da mamografia a partir dos 40 anos nas mulheres, sem, no entanto, especificar o tempo em anos da repetição do exame, o profissional fica exposto a questóes éticas e aos processos judiciais inerentes aos atendimentos desses paciente do SUS, realizados nos ambulatórios. Com essa vivência, o futuro médico terá sua base de atendimento e experiência consolidadas ainda no curso de Graduação em Medicina e apto para o mercado de trabalho neste setor. Portanto, o público alvo deste trabalho será a graduação de Medicina, porque este enfoque possibilitará uma atuação segura do médico de amanhã em seu benifício próprio, de seus pacientes, com diagnóstico precoce e tratamento menos agressivo possível, uma vez, que o câncer de mama atinge todas as faixas etárias, antes dos 50 e após os 65 anos de idade, com incidências diferentes principalmente naquelas pessoas sem antecedentes familiares, porque essa população é muito mais numerosa. E por outro lado, se não for solicitada a mamografia pelo médico naquelas pacientes após os 40 anos de idade, este profissional estará ao arrepio da lei e todas as suas consequencias. Porisso, a importância do ensino na graduação Médica para rastreamento do câncer inicial da mama em mulheres, como preconiza à lei, as Sociedades de Ginecologia e não vinculado ao programa de saúde do governo. Com essa aprendizagem o aluno de hoje evitará no futuro os dissabores dos processos judiciais e éticos, além de exercer uma medicina de melhor qualidade. Outrossim, neste contexto, o corpo discente da graduação de medicina deverá atentar para as mamas densas, independente da faixa etária, que à mamografia poderá não revelar imagens patológicas, sendo necessário a sua complementação com o exame de ultrassonografia mamária. Daí a importância desta ferramenta diagnóstica no rastreamento do câncer inicial da mama em mulheres e tendo como público alvo o alunato de graduação em medicina, para que seja aplicada na sua prática profissional num futuro próximo e que com essa prerformance garanta desempenho médico e diagnósticos precoces com cirurgias mais convervadoras, menos mutilantes e agressivas e uma sobrevida maior para o paciente. Então, já na graduação o corpo discente na disciplina de ginecologia poderá identificar várias imagens radiológicas à mamografia, aquilatando a sua gravidade ou as esclarecendo através do exame de ultrassonografia mamária que irá caracteriza-las como cística ou sólida e ainda acrescentar algumas informaçóes quanto a suspeita ou não de maliginidade, que somente será estabelicido com a biópsia e exame histopatológico. Portanto, essa ferramenta de ensino proprocionará aos graduandos de medicina uma visão ampla do rastreamento do câncer inicial da mama em todos os seus aspectos desde a anamnese do paciente até o exame histopatológico da peça cirúrgica e o procedimento adequado para o caso.

\section{CONCLUSÃO}

O controle do câncer de mama no Brasil representa um dos grandes desafios, de saúde pública. O diagnóstico tardio da doença e a não identificação de mulheres em situação de alto risco apontam para a necessidade de um programa que estimule o diagnóstico precoce da doença. A mamografia é a radiografia das mamas que permite a detecção precoce do câncer. É capaz de mostrar lesôes, em fase inicial, muito pequena (em milímetros) e que ao exame clinico das mamas não são identificadas quando menores que $1 \mathrm{~cm}$. E a ultrassonografia mamária é empregada nas mulheres com idade menor que 35 anos ou como complementação diagnóstica da mamografia. Por outro lado, alguns mastologistas solicitam a mamografia como rastreamento em mulheres assintomáticas e sem antecedentes de risco, a partir dos quarenta anos de idade, anualmente ou de dois em dois anos. Enquanto outros só os fazem a partir dos cinqüenta anos de idade com a mesma periodicidade. Isto, é até objeto de celeumas em Congressos Nacionais e Internacionais da Especialidade, sem se chegar a um consenso. Vale salientar que aqueles que optam pelo uso deste método diagnóstico após os cinqüenta anos de idade estão as margens e aos rigores da lei.

Esses fatores nos levaram a refletir sobre os aspectos da informação junto aos acadêmicos de medicina, uma vez que estes serão, em sua grande maioria, os responsáveis pela divulgação desta politica de governo, independentemente da especialidade a ser seguida no futuro.

\section{REFERÊNCIAS BIBLIOGRÁFICAS}

AMARAL, José Luiz Gomes do. Raio-X da Saúde no Brasil. Jornal da Febrasgo, ano 16, ed. 117, Agosto 2010, pag. 4-7.

BRASIL. Constituição (1988). Constituição da República Federativa do Brasil. Brasília: Senado, 1988.

Ministério da Saúde. Implantando o Viva Mulher Programa Nacional de Controle do Câncer do Colo do Útero e de Mama. Ministério da Saúde: Rio de Janeiro, 2001. 
. Ministério da Saúde. Instituto Nacional do Câncer. Controle do câncer de mama: documento de consenso [documento da Internet]. 2004 [citado 06 dez.2010].Disponível em:http://www.inca.gov.br/publicacoes/Consensointegra.

. Ministério da Saúde. Instituto Nacional do Câncer. Estimativa de câncer: incidência de câncer no Brasil [documento da Internet]. 2008 [citado $06 \mathrm{dez}$ 2010]. Disponível em: http://www.inca.gov.br/estimativa/2008.

- Ministério da Saúde. DATASUS. Informaçôes de saúde [Internet]. Brasília; 2009 [citado 2010 DEZ 05 ]. Disponível em: http://tabnet.datasus.gov.br/cgi/deftohtm. exe?sia/cnv/pauf.def

BRITO, Luciane Maria Oliveira et al . Conhecimento, prática e atitude sobre o autoexame das mamas de mulheres de uma cidade do Nordeste do Brasil. Rev. Bras. Ginecol. Obstet., Rio de Janeiro, v. 32, n. 5, May 2010 . Available from <http://www.scielo.br/scielo. Access on 06 Dec. 2010.

CARVALHO, Jair Antonio. Alimentação Saudável: Uma reflexão andragógica sobre a nutrição do adulto. Dissertação de mestratdo UNIFOA - 2009. 10 p.

CLAGNAN, Willian Simóes et al. Idade como fator independente de prognóstico no câncer de mama. Rev. Bras. Ginecol. Obstet., Rio de Janeiro, v. 30, n. 2, Feb. 2008. Available from <http://www.scielo.br/scielo. access on 06 Dec. 2010.

FELIX, Lizarda Maria de Carvalho, AOKI, Tsutomu, LIMA, Sonia Maria Rolim Rosa. Tratamento das ondas de calor em mulheres com câncer de mama. Rev. Feminina. [documento da Internet]. 2010 [citado $06 \mathrm{dez}$ 2010]. Disponível em: http://lildbi.bireme.br/lildbi/docsonline/ lilacs/20100500/1073.pdf

FIALHO, RF, MARTINS, WP, NASTRI, CO, FILHO, FM. Rastreamento de câncer de mama por imagem Rev. Feminina. [documento da Internet]. 2008 [citado $06 \mathrm{dez}$ 2010]. Disponível em: http://lildbi.bireme.br/lildbi/docsonline/lilacs/20100500/1073.pdf

HUGUET, Priscila Ribeiro et al . Qualidade de vida e sexualidade de mulheres tratadas de câncer de mama. Rev. Bras. Ginecol. Obstet., Rio de Janeiro, v. 31, n.2, Feb. 2009.Availablefrom<http://www.scielo.br/scielo. php?script=sci_arttext\&pid=S0100-72032009000200003\&lng=en\&nrm=iso $>$. access on 06 Dec. 2010.
Instituto Nacional do Câncer - INCA. Câncer de mama. [on line] [Acessado em 2010 dezembro 04]. Disponível em:< http:www.inca.gov.com.br>.

JÚNIOR, R. F., KOIFMAN, S., SANTOS, N. R. M. et al. Conhecimento e prática do auto-exame de mama. Revista da Associação Médica Brasileira, v.52, n.5, São Paulo set./out. 2006.

MARCHI, Ailton Augustinho; GURGEL, Maria Salete Costa. Adesão ao rastreamento mamográfico oportunístico em serviços de saúde públicos e privados. Rev. Bras. Ginecol. Obstet., Rio de Janeiro, v. 32, n. 4, Apr. 2010. Available from <http://www.scielo.br/scielo. Access on 06 Dec. 2010.

MECUM, Vade. LICC. Lei de introdução ao código civil. Decretolei 4.657/1942. 7aed. Atual. e ampl. - São Paulo: Saraiva, 2009.

NEVES, Nedy Maria Branco Cerqueira; SIQUEIRA, José Eduardo de. A bioética no atual Código de Ética Médica. Revista Bioética 2010; 18 (2): 439 - 50

OLIVEIRA, E.L; FREITAS-JUNIOR R; AFIUNE-NETO, A; MURTA, E.F, FERRO, J.E; MELO, A.F. Vascular calcifications seen on mammography: an independent factor 12 . indicating coronary artery disease. Clinics (São Paulo). 2009;64(8):763-7.

PAULINELLI, Régis Resende, MOREIRA, Marise Amaral Rebouças, FREITAS JUNIOR, Ruffo de. A Importância do Diagnóstico Precoce do Câncer de Mama Rev. Feminina. [documento da Internet]. 2004 [citado $06 \mathrm{dez}$ 2010]. Disponível em: http://lildbi.bireme.br/lildbi/docsonline/ lilacs/20100500/1073.pdf

RESENDE, Lúcio Márcio Perri de et al . Avaliação de microcalcificaçôes mamárias de acordo com as classificaçôes do Breast Imaging Reporting and Data System (BI-RADS TM) e de Le Gal. Rev. Bras. Ginecol. Obstet., Rio de Janeiro, v. 30, n. 2, Feb. 2008 .Available from < http://www. scielo.br/scielo. Access on 06 Dec. 2010

RODRIGUES, Monica Cristina de Souza; CALAS, Maria Julia Gregorio; NADRUZ, Eduardo. Mamografia: exame único, tecnologias diversas. Rev. Feminina. [documento da Internet]. 2008 [citado $06 \mathrm{dez}$ 2010]. Disponível em: http:// lildbi.bireme.br/lildbi/docsonline/lilacs/20100500/1073.pdf

\section{Endereço para Correspondência:}

Márcia Ribeiro Braz - marciabraz2009@gmail.com Faculdade de Medicina de Petrópolis - FMP/FASE Avenida Barão do Rio Branco, 905 - Petrópolis - RJ CEP: 25680-120 